\title{
Renal co-morbidity in patients with rheumatic diseases
}

\author{
Hans-Joachim Anders* and Volker Vielhauer
}

\begin{abstract}
Renal co-morbidity is common in patients with rheumatic disease based on regular assessment of serum and urine parameters of renal function. When patients present with both arthritis and renal abnormalities the following questions have to be addressed. Is kidney disease a complication of rheumatic disease or its management, or are they both manifestations of a single systemic autoimmune disease? Is rheumatic disease a complication of kidney disease and its management? How do rheumatic disease and kidney disease affect each other even when they are unrelated? The present review provides an overview of how to address these questions in daily practice.
\end{abstract}

\section{Introduction}

Rheumatic disease and kidney disease are both common in the general population. Rheumatologists are thus frequently exposed to patients with concomitant renal disease. In fact, $18 \%$ of rheumatology clinic patients were reported to have a glomerular filtration rate (GFR) of $60 \mathrm{ml} /$ minute or less as compared with the $5 \%$ reported within the general population [1]. When patients present with both, arthritis and kidney disease, the following questions have to be addressed. Is kidney disease a complication of rheumatic disease or its management, or are they both manifestations of a single systemic autoimmune disease? Is rheumatic disease a complication of kidney disease and its management? How do rheumatic disease and kidney disease affect each other even when they are unrelated? The present review addresses these questions and may help attending specialists, either rheumatologists or nephrologists, to manage patients with concomitant rheumatic disease and kidney disease.

*Correspondence: hjanders@med.uni-muenchen.de

Medizinische Poliklinik, Klinikum der Universität München - Innenstadt, Pettenkoferstraße 8a, 80336 Munchen, Germany
How to diagnose and monitor renal co-morbidity?

Renal co-morbidity may not be apparent in terms of signs and symptoms, so functional parameters must be routinely measured. Serum creatinine is the most commonly used parameter for renal excretory function and is therefore used to predict the GFR; for example, by applying the Modification of Diet in Renal Disease formula. Nowadays, the estimated GFR defines the stage of chronic kidney disease (CKD) and most laboratories routinely report the estimated GFR along with serum creatinine results [2]. The Modification of Diet in Renal Disease formula as well as other formulas used for GFR prediction, however, have not been validated to reliably predict GFR in patients with rheumatic diseases. In fact, the Modification of Diet in Renal Disease formula may underestimate renal function in patients with rheumatoid arthritis (RA) [3,4] or with lupus nephritis [5]. Concomitant elevations of blood urea nitrogen and uric acid imply renal excretory failure but only at an advanced stage of kidney damage.

Excretion is only one of the many renal functions (Table 1), and the aforementioned parameters can fail to indicate serious and potentially fatal kidney disease. For example, serum parameters may not indicate damage to the glomerular filtration barrier, which only becomes evident on urinalysis. Proteinuria and hematuria, as detected by urine dipstick analysis, is suggestive of glomerulonephritis (GN) - in systemic lupus erythematosus (SLE) or anti-neutrophil cytoplasmic antibody (ANCA) vasculitis, for example - and massive proteinuria is a hallmark of membranous lupus nephritis, amyloid-A amyloidosis or certain drug toxicities (Table 2). Dipstick urinalysis is routinely performed during the initial evaluation and monitoring visits by most rheumatologists [6], although their lack of accuracy to detect albuminuria advocates other methods [7]. A positive dipstick result at least requires further testing.

A number of tests can help to discriminate whether proteinuria originates either from overflow of serum proteins, glomerular damage or predominant tubulointerstitial damage. A simple screening test is quantitative urinary protein, albumin, and creatinine taken from a spot urine sample. Quantitative urinary protein will be 
Table 1. Renal functions and related clinical or laboratory parameters

\begin{tabular}{ll}
\hline Function & Clinical or laboratory parameter \\
\hline Excretion & Serum levels of creatinine, blood urea nitrogen, uric acid \\
Filtration barrier & Proteinuria as determined by dipstick test and/or by urinary protein/creatinine ratio, urinary albumin/creatinine ratio \\
& Hematuria as determined by dipstick test and/or microscopy \\
Sodium balance & Blood pressure and edema to be evaluated by clinical examination \\
Water balance & Serum sodium concentration, serum osmolarity \\
Acid-base status & Serum bicarbonate (and chloride) concentration \\
Renal hormones & Erythropoietin: hemoglobin level \\
& $1,25-(\mathrm{OH})_{2}$ vitamin D: serum calcium/phosphorus \\
& Concentrations, iPTH \\
\hline
\end{tabular}

iPTH, intact parathyroid hormone.

Table 2. Renal toxicities of drugs commonly used by rheumatologists

\begin{tabular}{ll}
\hline Renal manifestation & Drug \\
\hline Acute renal failure & $\begin{array}{l}\text { NSAIDs, cyclosporine overdose, } \\
\text { biphosphonates }\end{array}$ \\
Chronic renal failure & NSAIDs, cyclosporine overdose \\
Proteinuria/nephrotic syndrome & NSAIDs, gold \\
Sodium retention/edema/hypertension & NSAIDs \\
Arterial hypertension & Leflunomide, cyclosporine
\end{tabular}

NSAID, nonsteroidal anti-inflammatory drug.

measured by the salicylate method, which also detects immunoglobulin light chains that are missed by the dipstick analysis. If albuminuria accounts for most of the proteinuria, a glomerular disease can be anticipated. Vice versa, if albuminuria accounts only for a minor proportion, the proteinuria will originate from either protein overflow or tubular disease. Because urinary protein and albumin concentrations are confounded by fluid intake and water clearance, these parameters are divided by the urinary creatinine concentration to calculate the urinary protein/creatinine ratio or the urinary albumin/creatinine ratio. The spot urine albumin/creatinine ratio was already shown in 1983 to be a suitable surrogate parameter for the 24-hour urine protein excretion [8]. As a consequence, most institutions no longer collect 24-hour urine levels. Several studies document the accuracy of this approach; for example, for monitoring lupus nephritis $[9,10]$.

The kidney has more functions beyond toxin excretion and filtration - for example, salt and water homeostasis, acid-base balance, and hormone production. As such, an elevated blood pressure and edema suggest renal sodium retention with or without serum creatinine elevations. For example, a high blood pressure and edema may be the first clinical signs in a patient with nephrotic syndrome due to lupus nephritis, membranoproliferative GN in cryoglobulinemia or nonsteroidal anti-inflammatory drug (NSAID)-induced minimal change disease. The kidney produces three important regulators of homeostasis: renin, erythropoietin, and activated vitamin D. Regarding the latter, low serum calcium and elevated serum phosphorus must be recognized as a manifestation of CKD (that is, secondary hyperparathyroidism). Together, routine assessment should include serum creatinine with the estimated GFR, electrolytes, urine dipstick analysis, and blood pressure in all patients. The urinary protein/ creatinine ratio or the urinary albumin/creatinine ratio, blood urea nitrogen, uric acid, cystatin $\mathrm{C}$, serum bicarbonate, hemoglobin, serum calcium, phosphorus, and intact parathyroid hormone (iPTH) levels need to be monitored in CKD patients. Whether or not a kidney biopsy is required should be discussed with a nephrologist.

\section{Renal and rheumatic manifestations of systemic autoimmune disease}

Rheumatic diseases are systemic autoimmune diseases that preferentially affect joints and soft tissues, but the inflammatory process regularly involves solid organs, including the kidney (Table 3). In addition, toxicity of disease-modifying antirheumatic drugs (DMARDs) frequently affects the kidney. The attending specialist is left with the dilemma of these differential diagnoses that require either intensifying or reducing drug therapy (Table 2).

\section{Rheumatoid arthritis}

Owing to their high prevalence, RA and renal disease often coincide. The renal toxicity of antirheumatic drugs (for example, NSAID or cyclosporine toxicity), secondary renal disease induced by the chronic inflammatory process (especially renal amyloidosis) and, potentially, renal manifestations of the primary disease process, however, are important differential diagnoses.

In a prospective study of renal disease in 235 patients with early RA, 7\% developed persistent proteinuria and $6 \%$ presented elevated serum creatinine with or without 
Table 3. Renal manifestations of systemic diseases commonly seen by rheumatologists

\begin{tabular}{ll}
\hline Disease & Renal manifestation \\
\hline Rheumatoid arthritis & Mesangial GN, renal amyloidosis, membranous GN \\
Spondylarthropathy/psoriasis arthritis & Renal amyloidosis, IgA nephropathy \\
Systemic lupus erythematosus & Lupus nephritis: proliferative immune complex GN, membranous GN \\
Sjögren's syndrome & Interstitial nephritis with renal tubular acidosis (type 1) \\
ANCA vasculitis & Crescentic (pauci-immune) GN, interstitial nephritis \\
Giant cell arteritis & Renal artery stenosis \\
Immune complex disease & Immune complex GN \\
Diffuse cutaneous systemic scleroderma & Scleroderma renal crisis, crescentic GN (with myeloperoxidase ANCA), interstitial nephritis, chronic (ischemic) \\
& kidney disease \\
Sarcoidosis & Nephrocalcinosis, nephrolithiasis, granulomatous interstitial nephritis \\
Malignancy & Nephrotic syndrome (membranous GN, minimal change disease, focal glomerulosclerosis) \\
Diabetes & Chronic kidney disease: diabetic nephropathy \\
Hypertension & Chronic kidney disease: hypertensive nephropathy \\
\hline
\end{tabular}

ANCA, anti-neutrophil cytoplasmic antibody; GN, glomerulonephritis.

proteinuria during a 42-month observation period [11]. These abnormalities were mostly drug-related and reversible after discontinuation of the treatment. DPenicillamine and gold (but not methotrexate) were most often associated with proteinuria. Drug-induced proteinuria was associated with age over 50 years and elevation of C-reactive protein levels or the erythrocyte sedimentation rate. Seventeen percent of patients displayed microhematuria at study entry, with intermittent or persistent hematuria occurring in $42 \%$ of patients during the study period. Hematuria was again more common in patients older than 50 years and was associated with RA activity rather than treatment [11].

In the more recent Methotrexate and Renal Insufficiency (MATRIX) study, elevated serum creatinine values were found in $19 \%$ of prevalent RA patients. Among these, $20 \%$ were in stage 2 and 15\% were in stage 3 of CKD. Proteinuria, hematuria and leucocyturia were observed in $16 \%, 17 \%$ and $20 \%$ of the patients, respectively [12]. The causes of renal pathology were not determined in this study. In 1995, however, Helin and colleagues reported a retrospective analysis of renal biopsies from 110 RA patients in whom the clinical renal disease was probably due to antirheumatic therapy or RA itself [13]. The most common histopathologic finding was mesangial GN in $36 \%$, followed by amyloidosis (30\%) and membranous GN (17\%). Focal proliferative GN (4\%), minimalchange nephropathy (3\%), and acute interstitial nephritis (1\%) were less common. Amyloidosis was the most frequent finding in patients with nephrotic syndrome. In patients with isolated proteinuria, amyloidosis, membranous GN, and mesangial GN were almost equally common [13]. Similar results were reported in 1998 from Japan [14]. Novel DMARDs including biological drugs are more effective in suppressing systemic inflammation, however, and the incidence of renal amyloidosis has therefore declined [15]. Whether mesangial GN, which was frequently characterized by hematuria and glomerular deposition of IgM-containing immune complexes, is directly related to RA is still a matter for debate. In contrast, rheumatoid vasculitis, a rare but severe complication of chronic, erosive RA, may directly affect the kidneys. This vasculitis causes a necrotizing immune complex GN that requires intense immunosuppression $[16,17]$.

Regardless of the etiology, concurrent renal disease is a predictor of mortality in RA patients. Compared with controls the hazard ratio for mortality was 1.78 in one population-based cohort study from Finland [18]. In this study, nephropathy presenting with combined hematuria and proteinuria, proteinuria alone, microalbuminuria, or histologically confirmed amyloidosis was associated with increased mortality in RA patients, whereas mortality was within expected limits in those with isolated hematuria or mesangial GN [18].

\section{Spondyloarthritis and psoriatic arthritis}

Thirty-five percent of patients with ankylosing spondylitis display abnormal serum creatinine measurements or urinalyses, often resulting from glomerular deposition of amyloid A or IgA [19]. Drug-induced nephrotoxicities for example, interstitial nephritis or NSAID-associated membranous GN - are important differential diagnoses that deserve different therapeutic approaches. AmyloidA nephropathy requires aggressive control of the underlying rheumatic disease, whereas NSAID-induced interstitial nephritis or membranous GN requires cessation of these drugs. In the latter case, glucocorticoids or TNFblocking biologicals are alternative options for antiphlogistic therapy, and acetaminophen, metamizol/ 
dipyrone or opioids should be used as alternative analgesic medications. Importantly, amyloid-A nephropathy and NSAID-induced membranous GN may both present through nephrotic syndrome, requiring a renal biopsy to make a diagnosis. Patients with psoriatic arthritis and other spondyloarthritides (for example, reactive arthritis) may also present with IgA nephropathy or amyloid-A nephropathy. In psoriatic arthritis patients, elevation of serum creatinine values and/or proteinuria correlates with age, disease activity and duration [20].

\section{Systemic lupus erythematosus}

Lupus nephritis determines long-term morbidity and mortality in SLE because renal failure is associated with a large number of secondary and tertiary complications, as discussed in detail below. Lupus nephritis affects up to $50 \%$ of patients with SLE, and screening SLE patients for renal manifestations of lupus is therefore mandatory at each visit. Screening intervals might be longer in patients with discoid lupus because they experience lupus nephritis in less than 5\% of cases [21]. No reliable biomarker can predict the development of lupus nephritis in a non-nephritic patient to date, but the presence of U1snRNP antibodies, C1q antibodies, inherited complement deficiencies or high levels of anti-dsDNA antibodies are associated with a higher prevalence of nephritis in SLE patients.

Lupus nephritis can range from mild immune complex disease to diffuse proliferative GN as classified by the current International Society of Nephrology (ISN)/Renal Pthology Society (RPS) classification [22]. Immune complex deposition within the mesangium mainly activates mesangial cells and causes mesangioproliferative GN with hematuria and various degrees of proteinuria. The severity of renal disease correlates with the extent of immune complex deposits inside the glomerular mesangium and capillary network (ISN/RPS classes 1 to 4). When lupus immune complexes deposit outside the glomerular basement membrane, they mainly activate podocytes and cause large-scale proteinuria; that is, nephrotic syndrome (ISN/RPS class 5), which can occur together with any of the other classes. ISN/RPS class 6 represents glomerulosclerosis, a postinflammatory state of fibrotic scaring secondary to lupus nephritis without major inflammatory disease activity. Additional parameters indicate activity and chronicity of the lesions which may provide important clues for individual treatment strategies [22].

There are three main reasons that argue for performing a renal biopsy in SLE patients with abnormal urinalysis. First, several studies document that ISN/RPS class 3 and class 4 lupus nephritis can be present in patients with only minor urine abnormalities, such as microhematuria or a protein/creatinine ratio of 0.2 to 0.5 [23]. Second, only ISN/RPS classes 3 to 5 with relevant disease activity are at risk for developing renal failure and might benefit from immunosuppressive therapy. As such, a renal biopsy may not be needed to confirm the diagnosis of lupus nephritis, but to enforce mandatory therapy despite side effects. On the contrary, unnecessary treatments are avoided when biopsy results indicate renal involvement with low inflammatory activity. Rheumatologists (and nephrologists) have to ask the pathologist to provide all necessary information included in the ISN/RPS classification of lupus nephritis, especially the lupus activity index and chronicity index. This information will be provided by specialized renal pathologists. Third, SLE patients may suffer from kidney diseases other than lupus nephritis [23]; for example, interstitial nephritis or thrombotic microangiopathy as a manifestation of antiphospholid antibody syndrome.

\section{Other immune complex diseases}

Arthritis and nephritis are also common in other types of immune complex diseases such as postinfectious immune complex disease, Henoch-Schönlein purpura, cyroglobulinemia or endocarditis. While the latter example has its own diagnostic and treatment algorithms, all other types of immune complex disease may benefit from a renal biopsy, because the standard histological work-up of the obtained renal tissue often provides diagnostic clues to the underlying systemic disease. Examples include endocapillary GN in postinfectious immune complex disease, IgA nephropathy in Henoch-Schönlein purpura, and membranoproliferative $\mathrm{GN}$ in cyroglobulinemia.

Anti-neutrophil cytoplasmic antibody-associated vasculitis ANCA-associated vasculitis involves the glomerular capillaries leading to $\mathrm{GN}$ in up to $80 \%$ of Wegener's patients. Abnormal serum creatinine/cystatin $C$ levels and urinalysis indicate renal involvement. Urine microscopy displays dysmorphic erythrocytes, acanthocytes, and erythrocyte casts as a sign of destructive GN. On renal biopsy, ANCA-associated vasculitis typically presents as a pauci-immune (devoid of immune complexes), necrotizing and crescentic GN - which is why renal ANCA-associated vasculitis often presents as a rapid progressive disease with advanced stage of renal failure. Immunosuppressive therapy with cyclophosphamide and glucocorticoids must be initiated the same day to preserve renal function $[24,25]$. In addition, patients with end-stage renal disease should be treated with plasmapheresis to improve outcome [26].

\section{Panarteriitis nodosa and giant cell arteriitis}

Vasculitic involvement of intermediate-sized and large vessels in panarteriitis nodosa and giant cell arteriitis, respectively, may occasionally lead to renal functional impairment $[27,28]$. Inflammation and formation of 
microaneurysms in arteries of the renal parenchyma in panarteriitis nodosa or vasculitic stenosis of renal arteries and their branches in giant cell arteriitis will lead to chronic renal hypoperfusion and ischemia. Without involvement of the small glomerular capillaries these present clinically as progressive renal impairment and albuminuria, usually without gross hematuria and leukocyturia on urinalysis. Imaging studies rather than renal biopsy are required to confirm the diagnosis.

\section{Scleroderma}

Scleroderma can be complicated by a number of different forms of kidney disease [29]. The classical manifestation is scleroderma renal crisis, which represents a form of malignant hypertension with acute renal failure and potentially other organ manifestations due to excessive hypertension. Hypertensive crisis develops because the microvascular abnormalities in scleroderma cause renal hypoperfusion, triggering excessive release of renin that activates angiotensin. Immediate angiotensin blockade is therefore necessary. Renal crisis is an intriguing complication of diffuse cutaneous systemic sclerosis but affects only $2 \%$ of patients. Many more patients have ischemic nephropathy leading to slowly progressing CKD that is accompanied by hypertension and albuminuria. Blood pressure measurement and dipstick analysis are therefore mandatory at all routine check-ups of scleroderma patients. Normotensive acute renal failure should be evaluated for interstitial nephritis or ANCA vasculitis, a separate entity in scleroderma with poor outcome [30]. A renal biopsy is usually required to obtain the diagnosis in scleroderma patients with renal failure.

\section{Sarcoidosis}

Ectopic production of calcitriol, mainly by activated macrophages, causes hyperabsorption of dietary calcium in up to $50 \%$ of patients with sarcoidosis [31]. This excess calcium load leads to hypercalcuria, and in 2 to $20 \%$ of cases leads to hypercalcemia. The increased urinary excretion of calcium may cause nephrolithiasis, nephrocalcinosis, and polyuria. Nephrolithiasis has been reported in up to $14 \%$ of patients with sarcoidosis and may be the initial presenting feature of as well as the cause for renal failure in sarcoidosis patients [31,32]. Granulomatous interstitial nephritis is another typical renal manifestation of active diffuse sarcoidosis [33]. In this renal disease, urinalysis can be either normal or show sterile leukocyturia or mild tubular proteinuria that responds to glucocorticosteroids [33]. Sarcoidosis-related glomerular diseases are rare and their linking mechanisms are unknown [34]. A nephritic sediment with erythrocyte casts or heavy proteinuria should differentiate these from interstitial nephritis, although the diagnosis will be obtained by renal biopsy only.

\section{Renal and rheumatic manifestations of other systemic diseases \\ Paraneoplastic syndromes}

A variety of renal and musculoskeletal disorders can be associated with malignancy. Both types of disorder may occur concomitantly in individual patients, and differential diagnosis may be difficult, especially with regard to systemic autoimmune disease. Membranous GN, minimal change nephropathy and focal glomerulosclerosis are the most common renal manifestations of malignant tumors [35]. The predominant clinical finding in these conditions will be proteinuria with or without elevated serum creatinine. More rarely, membranoproliferative $\mathrm{GN}$, rapid progressive $\mathrm{GN}$, thrombotic microangiopathy, and interstitial renal disease have been described in isolated patients with solid tumors or hematologic malignancies. These paraneoplastic conditions may additionally be accompanied by tumor-associated arthralgias, oligoarthritis or polyarthritis, polymyalgia and dermatomyositis. More rarely, lupus-like syndromes, vasculitis and eosinophilic fasciitis may develop due to an underlying malignancy [36].

Establishing the correct diagnosis of a paraneoplastic syndrome rather than a systemic autoimmune disease with renal and rheumatic manifestation may be difficult, but is obviously of crucial importance for the patient's treatment and prognosis. These paraneoplastic syndromes must be differentiated from disease that directly results from malignancy. For example, multiple myeloma may cause renal damage due to amyloidosis, glomerular light/heavy chain deposition disease or cast nephropathy, as well as musculoskeletal pain due to local tumor growth. In addition, tumor lysis syndrome can cause uric acid nephropathy as well as crystal arthropathy. Finally, end-stage renal disease and proteinuria cause an elevated erythrocyte sedimentation rate, which should not be suspected as active rheumatic disease or occult malignancy [37,38].

\section{Diabetes mellitus}

Diabetic nephropathy is the most common cause of endstage renal disease in the western world. Glomerular sclerosis due to chronic hyperglycemia leads to proteinuria up to the nephrotic range, and is paralleled by a progressive rise in serum creatinine values. Diabetes may also cause musculoskeletal disorders, including diabetic cheiroarthropathy of the hands, and neuropathic Charcot arthropathy affecting the feet [39]. Other musculoskeletal complications seen in diabetic patients are Dupuytren's contracture and carpal tunnel syndrome, although in end-stage renal disease the latter may also be caused by dialysis-related amyloidosis due to local deposition of $\beta_{2}$ microglobulin (see below). Adhesive capsulitis of the shoulder and diffuse idiopathic skeletal hyperostosis are also associated with diabetes [39]. 


\section{Musculoskeletal manifestations of renal disease}

Mineral and bone disorders are a central complication of CKD (referred to as CKD-MBD), because renal failure impairs renal phosphorus clearance and the activation of 25-OH vitamin $\mathrm{D}$. The resulting hyperphosphatemia and hypocalcemia both induce secondary hyperparathyroidism, which is associated with two major consequences: bone mineral loss and extraosseous calcifications. Parathyroid hormone-induced bone mineral loss (that is, renal osteodystrophy) is often associated with osteoporosis due to immobilization during hemodialysis and due to CKD-associated systemic inflammation. Both increase the risk of fracture-related morbidity [40].

The most common complication of CKD-MBD is accelerated atherosclerosis leading to cardiovascular and cerebrovascular events. Extravascular calcifications in CKD-MBD also include chondrocalcinosis and pseudogout due to the precipitation of calcium pyrophosphate dihydrate crystals. The international Kidney Disease Improving Global Outcomes initiative guidelines are available for evidence-based CKD-MBD management and inform about the use of vitamin D analogs, phosphate binders, and calcium supplements [40]. The impaired renal uric acid clearance leads to hyperuricemia. Concomitant use of diuretics and/or cyclosporin is common and can further impair uric acid clearance and increase the risk of gout attacks. Advanced CKD represents a state of systemic immunosuppression that can alter the clinical presentation of autoinflammatory diseases like gout. Acute gout attacks can be mitigated in CKD, and symptomatic patients should be examined for signs of chronic gout [41]. Treating crystal arthropathy is problematic in CKD as drugs commonly used to treat acute gout attacks, such as NSAIDs, should be avoided in CKD patients (Table 4). Colchicine should also be avoided because the narrow therapeutic range makes appropriate dose adjustments difficult [41]. We use $20 \mathrm{mg}$ prednisolone for 3 to 5 days to control acute gout attacks and advise patients to start steroids immediately upon first symptoms of an attack. The central role of IL- $1 \beta$ in the pathophysiology of crystal arthritis suggests that IL-1 inhibitors are efficient in CKD patients $[42,43]$. Interval control of hyperuricemia and the treatment of chronic gout require dose adjustments of allopurinol or benzbromarone (when GFR $>50 \mathrm{ml} /$ minute $/ 1.73 \mathrm{~m}^{2}$ ). Some reports propose febuxostat, uricase or anakinra as alternative treatments for gout, which may offer advantages for CKD patients $[41,43,44]$.

Carpal tunnel syndrome, bone cysts, and destructive spondyloarthropathy or joint arthropathy used to be classical complications for end-stage kidney disease patients affected by dialysis-related amyloidosis, but their prevalence declined upon broad use of high-flux dialysers [45]. Impaired clearance of small proteins like $\beta_{2}$-microglobulin leads to $\beta$-sheet amyloid fibrils. Symptomatic amyloidosis rarely developed before 5 years of hemodialysis but the use of high-flux dialysis filters has recently delayed the onset of dialysis-related amyloidosis. $\beta_{2}$-Microglobulin amyloid deposition in bone, joints, and tendons causes pain, stiffness, and arthritis. Bone cysts can cause fractures and tendon deposits cause tears in ligaments and tendons [45].

\section{Implications of unrelated chronic kidney disease in the rheumatic patient}

Patients with impaired renal function have a substantial increase in cardiovascular risk that can be explained only in part by an increase in traditional risk factors such as hypertension and diabetes. It is now well recognized that CKD alone is an independent risk factor for cardiovascular complications $[46,47]$. International guidelines thus stress the need for preventing and treating cardiovascular risk factors in patients with CKD, as has been proposed for RA or SLE patients [48-51]. It is very likely that patients with rheumatic diseases and concomitant CKD are threatened by additive proatherogenic effects, and such individuals should hence be regarded as high-risk patients for cardiovascular complications that deserve rigorous control of their rheumatic disease activity. Several DMARDs cannot be used in CKD patients, however, due to their inherent nephrotoxicity. For example, NSAIDs (including cyclooxygenase-2 inhibitors) can cause acute deterioration of renal function, which is more common particularly in older patients, in CKD patients or in heart failure patients, and in states of volume depletion, because renal blood flow is dependent on renal prostaglandin synthesis [52,53]. Glucocorticoids, acetaminophen, metamizol/dipyrone or opioids can replace NSAIDs in CKD patients. Cyclosporine A is another potentially nephrotoxic DMARD. Cyclosporine causes vasoconstriction of the afferent and efferent glomerular arterioles, which leads to reductions in renal blood flow and the GFR. Chronic administration of cyclosporine can cause obliterative arteriolopathy, ischemic scarring, tubular atrophy and progressive loss of renal function. Risk factors include high dose, increased age and pre-existing CKD [54]. Cyclosporine is hence contraindicated in RA patients with renal dysfunction according to international guidelines $[55,56]$.

Renal insufficiency also impairs the excretion of some DMARDs, which increases (nonrenal) toxicity. For example, methotrexate is not nephrotoxic per se but it is excreted via the kidneys, and therefore accumulates and is increasingly toxic along the stages of CKD $[57,58]$. Some authors advocate dose reductions in CKD patients, but this can be dangerous because declines in the GFR can always happen with incident fluid losses - for example, through vomiting, diarrhea, use of diuretics or 
Table 4. Treatment of crystal arthropathies in chronic kidney disease and dialysis patients

\begin{tabular}{|c|c|c|}
\hline Drug & $\begin{array}{l}\text { CKD stage } 3 \text { and stage } 4 \text { patients } \\
\text { (GFR } 15 \text { to } 59 \mathrm{ml} / \text { minute } / 1.73 \mathrm{~m}^{2} \text { ) }\end{array}$ & $\begin{array}{l}\text { Dialysis patients } \\
\left(\mathrm{GFR}<15 \mathrm{ml} / \mathrm{minute} / 1.73 \mathrm{~m}^{2}\right)\end{array}$ \\
\hline \multicolumn{3}{|c|}{ Pain control in acute gout attack } \\
\hline NSAIDs & Avoid when possible or use with great caution & May be used with caution \\
\hline Colchicine & $\begin{array}{l}\text { Avoid when possible or use with great caution (for example, } \\
0.5 \mathrm{mg} \text { three times per week) }\end{array}$ & Avoid whenever possible \\
\hline Glucocorticoids & Short-term use possible (for example, 20 mg/day) & Short-term use possible (for example, 20 mg/day) \\
\hline Anakinra & Effective (100 mg/day for 3 days) but yet limited data published & Not evaluated \\
\hline \multicolumn{3}{|l|}{ Maintenance therapy } \\
\hline Allopurinol & Reduce dose to 100 to $150 \mathrm{mg}$ or avoid & Avoid \\
\hline Colchicine & $\begin{array}{l}\text { Avoid when possible or use with great caution (for example, } \\
0.5 \mathrm{mg} \text { three times per week) }\end{array}$ & Avoid whenever possible \\
\hline Anakinra & Not evaluated & Effective in case reports (100 mg three times per week) \\
\hline Febuxostat & Safe at GFR $>30 \mathrm{ml} /$ minute $/ 1.73 \mathrm{~m}^{2}$ & Not evaluated \\
\hline Benzbromarone & Probably ineffective at GFR $<50 \mathrm{ml} /$ minute & Ineffective \\
\hline
\end{tabular}

CKD, chronic kidney disease; GFR, glomerular filtration rate; NSAID, nonsteroidal anti-inflammatory drug.

NSAIDs, sweating or fever - which further impair methotrexate clearance without a physician even being consulted. In view of the potential lethal side effects and usually available alternative treatment regimens (for example, leflunomide or biologicals), methotrexate should generally be avoided in CKD patients. Antimalarials, sulfasalazine, and azathioprine are only partially excreted by the kidneys, and therefore dose reductions were proposed at GFR $<50 \mathrm{ml} /$ minute (for details see $[59,60])$.

Some DMARDs have specific side effects that add to the cardiovascular risk of patients with rheumatic disease. For example, cyclosporine or leflunomide often increases blood pressure, which adds blood pressure monitoring and blood pressure control $(<130 / 80 \mathrm{mmHg})$ to the responsibilities of the rheumatologist [49]. A typical side effect of tacrolimus and tocilizumab is hyperlipoproteinemia, which must be controlled by appropriate lipid-lowering therapy to levels below 70 to $100 \mathrm{mg} / \mathrm{dl}$ in rheumatic patients with CKD, most commonly with statins [48].

Anemia in patients with active rheumatic disease and renal insufficiency can be inflammation related and/or CKD related. Differential diagnosis may be difficult in individual patients, but persistent anemia despite control of inflammatory disease activity suggests a renal cause. These patients should receive iron supplementation and erythropoiesis-stimulating agents according to current guidelines [61].

It is also of note that the guidelines for the prevention of glucocorticoid-induced osteoporosis - that is, supplementation of calcium and vitamin D or biphosphonate therapy - are not validated and therefore do not apply to rheumatic patients with CKD. Bone metabolism in CKD patients is totally different from that in nonrenal patients with its own needs for prophylaxis and therapeutic interventions. In addition, the use of biphosphonates is mostly contraindicated in the CKD patient as it can increase the risk of adynamic bone disease and the risk of biphosphonate-associated nephrotoxicity [62].

Finally, diagnostic procedures such as computed tomography or magnetic resonance imaging involving iodine-containing or gadolinium-containing contrast agents, respectively, must be used with great caution in CKD patients of stage 4 and stage 5 . Radiocontrast agents often cause radiocontrast nephropathy that is associated with an increase of overall mortality, especially in CKD patients. If not avoidable, preventive measures such as discontinuation of diuretics and prehydration are mandatory to minimize the risk of radiocontrast nephropathy [63]. The potential benefits of prophylactic $N$-acetylcysteine for the prevention of radiocontrast nephropathy remain under debate. Nephrogenic systemic fibrosis is a rare but devastating complication in patients with endstage renal disease that may develop after administration of linear gadolinium chelate-containing contrast agents [64]. An active inflammatory process at the time of gadolinium exposure is an important risk factor for the subsequent development of this condition. Magnetic resonance imaging using linear gadolinium chelates of patients with active rheumatic disease and end-stage renal disease must therefore be avoided, and cyclic gadolinium chelates may be preferred in stage 4 CKD patients.

\section{Conclusion}

Patients with rheumatic diseases must be routinely monitored by blood and urinary parameters for 


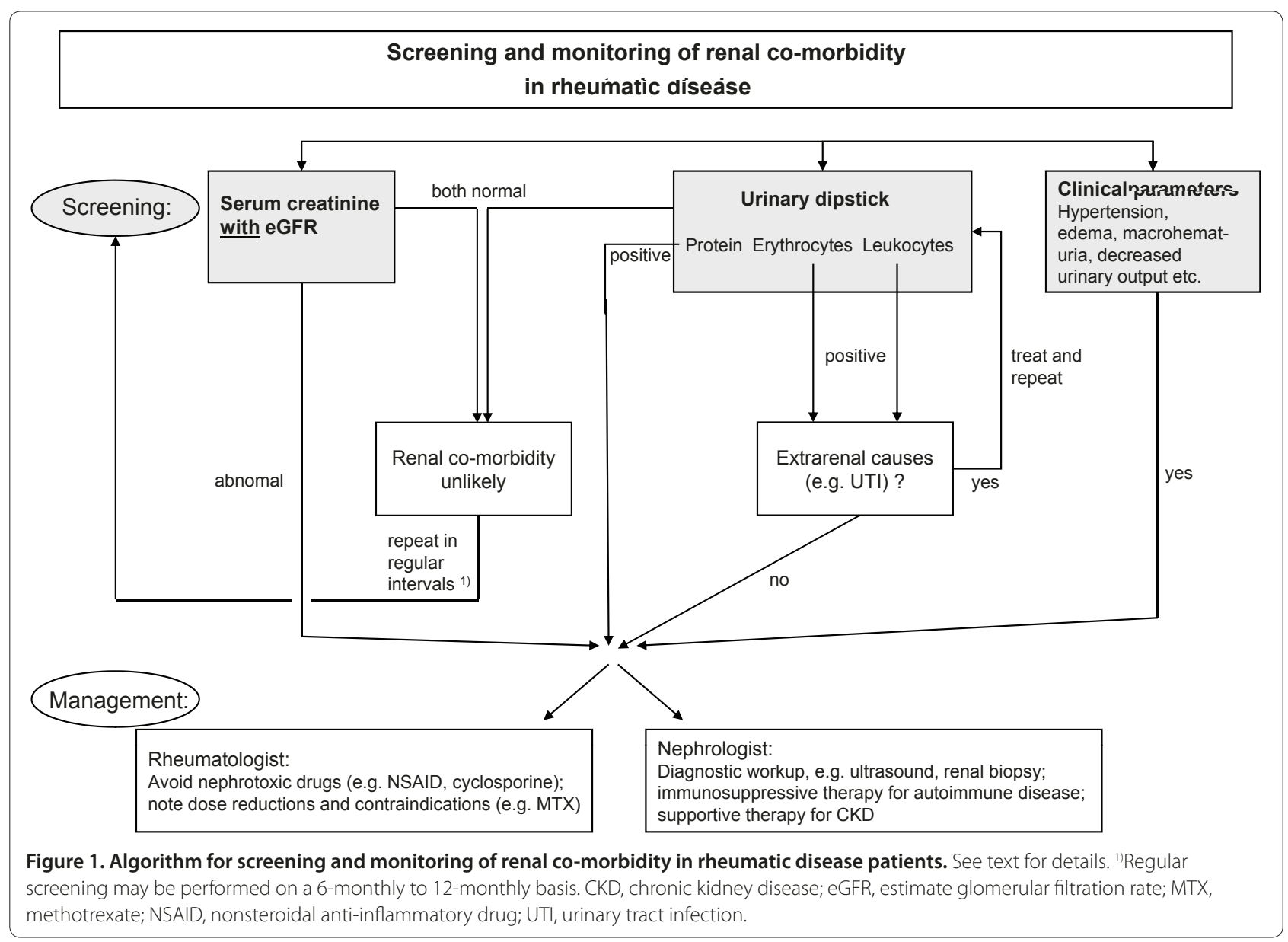

concomitant CKD, which can be either a manifestation of the same systemic disease, a side effect of DMARD therapy, or an unrelated renal disease (Figure 1). All three entities affect the diagnostic and therapeutic strategies of the rheumatologist. It should therefore be left to the expertise of the nephrologist to determine whether renal biopsy is required for the diagnostic work-up. Patients should be subdivided into those needing more intense immunosuppressive treatment because of their kidney disease and those needing dose reduction or modification of their medication because of their kidney disease. Ideally, patients with rheumatic diseases and renal comorbidity should be managed through close collaboration between a rheumatologist and a nephrologist.

This article is part of the series Comorbid conditions in subjects with rheumatic diseases, edited by Daniel Aletaha and Thomas Dörner. Other articles in this series can be found at http://arthritis-research.com/series/comorbid

\section{Abbreviations}

ANCA, anti-neutrophil cytoplasmic antibody; CKD, chronic kidney disease; DMARD, disease-modifying antirheumatic drug; GFR, glomerular filtration rate; GN, glomerulonephritis; IL, interleukin; iPTH, intact parathyroid hormone; ISN,
International Society of Nephrology; MBD, mineral and bone disorders; NSAID, nonsteroidal anti-inflammatory drug; RA, rheumatoid arthritis; RPS, Renal Pathology Society; SLE, systemic lupus erythematosus; TNF, tumor necrosis factor.

\section{Competing interests}

The authors declare that they have no competing interests.

Published: 29 June 2011

\section{References}

1. Hill AJ, Thomson RJ, Hunter JA, Traynor JP: The prevalence of chronic kidney disease in rheumatology outpatients. Scott Med J 2009, 54:9-12.

2. National Kidney Foundation: $\mathrm{K} / \mathrm{DOQI}$ clinical practice guidelines for chronic kidney disease: evaluation, classification, and stratification. Am J Kidney Dis 2002, 39(2 Suppl 1):S1-S266.

3. Anders HJ, Rihl M, Loch O, Schattenkirchner M: Prediction of creatinine clearance from serum creatinine in patients with rheumatoid arthritis: comparison of six formulae and one nomogram. Clin Rheumatol 2000, 19:26-29.

4. Anders HJ, Rihl M, Vielhauer V, Schattenkirchner M: Assessment of renal function in rheumatoid arthritis: validity of a new prediction method. J Clin Rheumatol 2002, 8:130-133.

5. Kasitanon N, Fine DM, Haas M, Magder LS, Petri M: Estimating renal function in lupus nephritis: comparison of the Modification of Diet in Renal Disease and Cockcroft Gault equations. Lupus 2007, 16:887-895.

6. Siedner MJ, Christopher-Stine L, Astor BC, Gelber AC, Fine DM: Screening for proteinuria in patients with lupus: a survey of practice preferences among American rheumatologists. J Rheumatol 2007, 34:973-977.

7. Siedner MJ, Gelber AC, Rovin BH, McKinley AM, Christopher-Stine L, Astor B, 
Petri M, Fine DM: Diagnostic accuracy study of urine dipstick in relation to 24-hour measurement as a screening tool for proteinuria in lupus nephritis. J Rheumatol 2008, 35:84-90.

8. Ginsberg JM, Chang BS, Matarese RA, Garella S: Use of single voided urine samples to estimate quantitative proteinuria. NEng/ J Med 1983, 309:1543-1546.

9. Salesi M, Karimifar M, Farajzadegan Z, Esalatmanesh K, Khosravi S, Fallahi P, Akbarian $\mathrm{M}$ : The protein-creatinine ratio in spot morning urine samples and 24-h urinary protein excretion in patients with systemic lupus erythematosus. Rheumatol Int 2009, 29:503-507.

10. Leung YY, Szeto CC, Tam LS, Lam CW, Li EK, Wong KC, Yu SW, Kun EW: Urine protein-to-creatinine ratio in an untimed urine collection is a reliable measure of proteinuria in lupus nephritis. Rheumatology (Oxford) 2007, 46:649-652.

11. Koseki Y, Terai C, Moriguchi M, Uesato M, Kamatani N: A prospective study of renal disease in patients with early rheumatoid arthritis. Ann Rheum Dis 2001, 60:327-331.

12. Karie S, Gandjbakhch F, Janus N, Launay-Vacher V, Rozenberg S, Mai Ba CU, Bourgeois P, Deray G: Kidney disease in RA patients: prevalence and implication on RA-related drugs management: the MATRIX study. Rheumatology (Oxford) 2008, 47:350-354.

13. Helin HJ, Korpela MM, Mustonen JT, Pasternack Al: Renal biopsy findings and clinicopathologic correlations in rheumatoid arthritis. Arthritis Rheum 1995, 38:242-247.

14. Nakano M, Ueno M, Nishi S, Shimada H, Hasegawa H, Watanabe T, Kuroda T, Sato T, Maruyama Y, Arakawa M: Analysis of renal pathology and drug history in 158 Japanese patients with rheumatoid arthritis. Clin Nephrol 1998, 50:154-160.

15. Immonen K, Finne P, Gronhagen-Riska C, Pettersson T, Kautiainen H, Hakala $\mathrm{M}:$ Steep decline in the incidence of renal replacement therapy for amyloidosis associated with inflammatory rheumatic diseases. Scand J Rheumatol 2009, 38:403.

16. Scott DG, Bacon PA, Elliott PJ, Tribe CR, Wallington TB: Systemic vasculitis in a district general hospital 1972-1980: clinical and laboratory features, classification and prognosis of 80 cases. Q J Med 1982, 51:292-311.

17. Scott DG, Bacon PA: Intravenous cyclophosphamide plus methylprednisolone in treatment of systemic rheumatoid vasculitis. Am J Med 1984, 76:377-384

18. Sihvonen S, Korpela M, Mustonen J, Laippala P, Pasternack A: Renal disease as a predictor of increased mortality among patients with rheumatoid arthritis. Nephron Clin Pract 2004, 96:c107-c1 14.

19. Vilar MJ, Cury SE, Ferraz MB, Sesso R, Atra E: Renal abnormalities in ankylosing spondylitis. Scand J Rheumatol 1997, 26:19-23.

20. Alenius GM, Stegmayr BG, Dahlqvist SR: Renal abnormalities in a population of patients with psoriatic arthritis. Scand J Rheumatol 2001, 30:271-274

21. Tebbe B, Mansmann U, Wollina U, Auer-Grumbach P, Licht-Mbalyohere A, Arensmeier M, Orfanos CE: Markers in cutaneous lupus erythematosus indicating systemic involvement. A multicenter study on 296 patients. Acta Derm Venereol 1997, 77:305-308.

22. Weening JJ, D'Agati VD, Schwartz MM, Seshan SV, Alpers CE, Appel GB, Balow JE, Bruiin JA, Cook T, Ferrario F, Fogo AB, Ginzler EM, Hebert L, Hill G, Hill P, Jennette JC, Kong NC, Lesavre P, Lockshin M, Looi LM, Makino H, Moura LA, Nagata M: The classification of glomerulonephritis in systemic lupus erythematosus revisited. J Am Soc Nephrol 2004, 15:241-250

23. Christopher-Stine L, Siedner M, Lin J, Haas M, Parekh H, Petri M, Fine DM Renal biopsy in lupus patients with low levels of proteinuria. J Rheumatol 2007, 34:332-335.

24. Hoffman GS, Kerr GS, Leavitt RY, Hallahan CW, Lebovics RS, Travis WD, Rottem M, Fauci AS: Wegener granulomatosis: an analysis of 158 patients. Ann Intern Med 1992, 116:488-498.

25. de Groot K, Harper L, Jayne DR, Flores Suarez LF, Gregorini G, Gross WL, Luqmani R, Pusey CD, Rasmussen N, Sinico RA, Tesar V, Vanhille P, Westman K, Savage CO; EUVAS (European Vasculitis Study Group): Pulse versus daily oral cyclophosphamide for induction of remission in antineutrophil cytoplasmic antibody-associated vasculitis: a randomized trial. Ann Intern Med 2009, 150:670-680.

26. Jayne DR, Gaskin G, Rasmussen N, Abramowicz D, Ferrario F, Guillevin L, Mirapeix E, Savage CO, Sinico RA, Stegeman CA, Westman KW, van der Woude FJ, de Lind van Wijngaarden RA, Pusey CD; European Vasculitis Study Group: Randomized trial of plasma exchange or high-dosage methylprednisolone as adjunctive therapy for severe renal vasculitis. J Am
Soc Nephrol 2007, 18:2180-2188.

27. Medvedev G, Al-Shamari AE, Copland MA, Magil AB: Isolated renal giant cell arteritis. Am J Kidney Dis 2002, 40:658-661.

28. Bakkaloglu SA, Ekim M, Tumer N, Tulunay O, Ozer T: Severe renal impairment in the case of classic polyarteritis nodosa. Pediatr Nephrol 2001, 16:148-150.

29. Penn H, Denton CP: Diagnosis, management and prevention of scleroderma renal disease. Curr Opin Rheumatol 2008, 20:692-696.

30. Anders HJ, Wiebecke B, Haedecke C, Sanden S, Combe C, Schlondorff D: MPO-ANCA-positive crescentic glomerulonephritis: a distinct entity of scleroderma renal disease? Am J Kidney Dis 1999, 33:e3.

31. Muther RS, McCarron DA, Bennett WM: Renal manifestations of sarcoidosis. Arch Intern Med 1981, 141:643-645.

32. Singer FR, Adams JS: Abnormal calcium homeostasis in sarcoidosis. N Engl J Med 1986, 315:755-757.

33. Rajakariar R, Sharples EJ, Raftery MJ, Sheaff M, Yaqoob MM: Sarcoid tubulointerstitial nephritis: long-term outcome and response to corticosteroid therapy. Kidney Int 2006, 70:165-169.

34. Göbel U, Kettritz R, Schneider W, Luft F: The protean face of renal sarcoidosis. J Am Soc Nephrol 2001, 12:616-623.

35. Birkeland SA, Storm HH: Glomerulonephritis and malignancy: a populationbased analysis. Kidney Int 2003, 63:716-721

36. Racanelli V, Prete M, Minoia C, Favoino E, Perosa F: Rheumatic disorders as paraneoplastic syndromes. Autoimmun Rev 2008, 7:352-358.

37. Abu-Romeh SH, Johny KV: The erythrocyte sedimentation rate in end-stage renal disease [letter]. Am J Kidney Dis 1988, 12:169.

38. Liverman PC, Tucker FL, Bolton WK: Erythrocyte sedimentation rate in glomerular disease: association with urinary protein. Am J Nephrol 1988 8:363-367.

39. Kim RP: The musculoskeletal complications of diabetes. Curr Diab Rep 2002, 2:49-52.

40. Miller PD: Diagnosis and treatment of osteoporosis in chronic renal disease. Semin Nephrol 2009, 29:144-155.

41. Gaffo AL, Saag KG: Management of hyperuricemia and gout in CKD. Am J Kidney Dis 2008, 52:994-1009.

42. So A, De Smedt T, Revaz S, Tschopp J: A pilot study of IL-1 inhibition by anakinra in acute gout. Arthritis Res Ther 2007, 9:R28.

43. Announ N, Palmer G, Guerne PA, Gabay C: Anakinra is a possible alternative in the treatment and prevention of acute attacks of pseudogout in endstage renal failure. Joint Bone Spine 2009, 76:424-426.

44. De Angelis S, Noce A, Di Renzo L, Cianci R, Naticchia A, Giarrizzo GF, Giordano F, Tozzo C, Splendiani G, De Lorenzo A: Is rasburicase an effective alternative to allopurinol for management of hyperuricemia in renal failure patients? A double blind-randomized study. Eur Rev Med Pharmacol Sci 2007, 11:179-184.

45. Yamamoto S, Kazama JJ, Narita I, Naiki H, Gejyo F: Recent progress in understanding dialysis-related amyloidosis. Bone 2009, 45(Suppl 1):S39-S42.

46. Keith DS, Nichols GA, Gullion CM, Brown JB, Smith DH: Longitudinal followup and outcomes among a population with chronic kidney disease in a large managed care organization. Arch Intern Med 2004, 164:659-663.

47. Foley RN, Murray AM, Li S, Herzog CA, McBean AM, Eggers PW, Collins A. Chronic kidney disease and the risk for cardiovascular disease, renal replacement, and death in the United States Medicare population, 1998 to 1999. J Am Soc Nephrol 2005, 16:489-495,

48. National Kidney Foundation: K/DOQI clinical practice guidelines for management of dyslipidemias in patients with kidney disease. Am J Kidney Dis 2003, 41 (4 Suppl 3):-IV, S1-S91.

49. National Kidney Foundation: K/DOQI clinical practice guidelines on hypertension and antihypertensive agents in chronic kidney disease. Am J Kidney Dis 2004, 43(5 Suppl 1):S1-S290.

50. National Kidney Foundation: KDOQI clinical practice guidelines and clinical practice recommendations for diabetes and chronic kidney disease. Am J Kidney Dis 2007, 49(2 Suppl 2):S12-S154.

51. Abboud H, Henrich WL: Clinical practice. Stage IV chronic kidney disease. NEngl J Med 2010, 362:56-65.

52. Whelton A: Nephrotoxicity of nonsteroidal anti-inflammatory drugs: physiologic foundations and clinical implications. Am J Med 1999, 106:13S-24S.

53. Hörl WH: Nonsteroidal anti-inflammatory drugs and the kidney. Pharmaceuticals 2010, 3:2291-2321.

54. Feutren G, Mihatsch MJ: Risk factors for cyclosporine-induced nephropathy 
in patients with autoimmune diseases. International Kidney Biopsy Registry of Cyclosporine in Autoimmune Diseases. N Engl J Med 1992, 326:1654-1660

55. Panayi GS, Tugwell P: The use of cyclosporin A microemulsion in rheumatoid arthritis: conclusions of an international review. Br J Rheumatol 1997, 36:808-811.

56. Cush JJ, Tugwell P, Weinblatt M, Yocum D: US consensus guidelines for the use of cyclosporin A in rheumatoid arthritis. J Rheumatol 1999, 26:1176-1186.

57. Gutierrez-Urena S, Molina JF, Garcia CO, Cuellar ML, Espinoza LR: Pancytopenia secondary to methotrexate therapy in rheumatoid arthritis. Arthritis Rheum 1996, 39:272-276.

58. Cheung KK, Chow KM, Szeto CC, Tai MH, Kwan BC, Li PK: Fatal pancytopenia in a hemodialysis patient after treatment with low-dose methotrexate. $J$ Clin Rheumatol 2009, 15:177-180.

59. Aronoff GR, Berns JS, Brier ME, Golper TA, Morrison G, Singer I, Swan SK, Bennett WM: Drug Prescribing in Renal Failure. Dosing Guidelines for Adults. 4th edition. Philadelphia, PA: American College of Physicians-American Society of Internal Medicine; 1999
60. Swarup A, Sachdeva N, Schumacher HR, Jr: Dosing of antirheumatic drugs in renal disease and dialysis. J Clin Rheumatol 2004, 10:190-204.

61. National Kidney Foundation: KDOQI clinical practice guideline and clinical practice recommendations for anemia in chronic kidney disease: 2007 update of hemoglobin target. Am J Kidney Dis 2007, 50:471-530.

62. Kidney Disease: Improving Global Outcomes (KDIGO) CKD-MBD Work Group: KDIGO clinical practice guideline for the diagnosis, evaluation, prevention, and treatment of Chronic Kidney Disease-Mineral and Bone Disorder (CKD-MBD). Kidney Int Supp/ 2009, 113:S1-S130.

63. Navaneethan SD, Singh S, Appasamy S, Wing RE, Sehgal AR: Sodium bicarbonate therapy for prevention of contrast-induced nephropathy: a systematic review and meta-analysis. Am J Kidney Dis 2009, 53:617-627.

64. Bardin T, Richette P: Nephrogenic systemic fibrosis. Curr Opin Rheumatol 2010, 22:54-58

doi:10.1186/ar3256

Cite this article as: Anders $\mathrm{H}-\mathrm{J}$, Vielhauer V: Renal co-morbidity in patients with rheumatic diseases. Arthritis Research \& Therapy 2011, 13:222. 